Palabra Clave (La Plata), octubre 2017, vol. 7, n 1, e034. ISSN 1853-9912

Universidad Nacional de La Plata.

Facultad de Humanidades y Ciencias de la Educación.

Departamento de Bibliotecología

\title{
Instrumento para la medición de la ciencia y la tecnología en la gestión de la información institucional. Caso de estudio
}

\author{
Measuring instrument for science and technology in \\ managing institutional information. Case study
}

\section{Soleidy Rivero Amador *, Maidelyn Díaz Pérez **, María José López- Huertas $* * *$, Reinaldo Javier Rodriguez Font $* * * *$}

* Universidad de Pinar del Río. Facultad de Ciencias Económicas y Empresariales. Vicedecana de Investigación y Posgrado, Cuba, ** Universidad de Pinar del Río. Departamento de Publicaciones y Grupo de Gestión de Información y Conocimiento (proGINTEC), Cuba, *** Universidad de Granada. Departamento de Bibliotecología, España, **** Universidad de Pinar del Río. Grupo de Gestión de Información y Conocimiento (proGINTEC), Cuba | soly@upr.edu.cu, maideyn@upr.edu.cu,mjlopez@ugr.es, rjfont@upr.edu.cu

\section{PALABRAS CLAVE}

\section{Curriculum vitae}

Indicadores de ciencia y tecnología

Sistemas de información curricular

Gestión de información

Gestión del conocimiento

Universidades

\section{KEYWORDS}

\section{Curriculum vitae}

Science and technology

indicators

Curricular information systems

Information management

Knowledge management

University

\section{RESUMEN}

En los últimos 10 años, los Sistemas de Información Curricular se han convertido en herramientas significativas en la aplicación de instrumentos de medición de la gestión de la ciencia y la tecnología a nivel institucional y regional. La presente investigación centra su atención en el desarrollo de un instrumento de medición para la gestión de la ciencia y la tecnología a nivel institucional; la propuesta se auxilia del análisis empírico de esta actividad en la Universidad de Pinar del Río (Cuba). Se utiliza como herramienta el Sistema Integral de Gestión de Información y Conocimiento institucional (CV-UPR), desarrollado por especialistas de esta Universidad. La investigación parte de la identificación de los referentes teóricos y conceptuales que sustentan la propuesta, mediante el método del análisis documental. Además se auxilia del método empírico de la observación, y de las entrevistas y encuestas como técnicas empíricas. El instrumento que se obtiene ofrece valores puntuales y además realiza comparaciones entre un conjunto de variables identificadas. Los análisis evaluativos realizados, mediante el cálculo de un sistema de indicadores, permitieron identificar las capacidades de la institución para la generación, diseminación y valoración de la información y el conocimiento institucional en el proceso de gestión de la ciencia y la tecnología, a nivel institucional.

Recibido: 29 de mayo de 2017 | Aceptado: 29 de agosto de 2017 | Publicado: 9 de octubre de 2017

Cita sugerida: Rivero Amador, S., Díaz Pérez, M., López-Huertas, M. J. y Rodríguez Font, R. J. (2017). Instrumento para la medición de la ciencia y la tecnología en la gestión de la información institucional. Caso de estudio. Palabra Clave (La Plata),7(1), e034. https://doi.org/10.24215/18539912e034 


\section{Introducción}

En la actualidad, se pueden percibir una gran diversidad de formatos que diseminan la información y además retienen el conocimiento explícito de la propia sociedad, ya sea a nivel personal, grupal, organizacional o regional. Este fenómeno social precisa de instrumentos, métodos y políticas institucionales y regionales para lograr el procesamiento de la información comunicándola, haciéndola accesible (mediante la recuperación), organizándola, e incluso sometiéndola a mediación informática.

Casi a las puertas de la segunda década del siglo XXI los investigadores interactúan con nuevas realidades y dimensiones comunicativas derivadas del propio desarrollo de la digitalización de la información (Arango Forero, 2013). Desde esta perspectiva, toda organización requiere de un flujo constante de información organizada y oportuna que logre coordinar sus acciones operativas y mantener informados a sus decisores y miembros de la organización. Esta necesidad es un reflejo del desempeño estratégico de la organización (Andreu, Ricart y Valor, 1996). Al respecto, los Sistemas de Información (SI) han logrado protagonismo en la praxis de la gerencia organizacional. Su contribución está enmarcada en perfeccionar la toma de decisiones institucionales, contribuir en el desempeño exitoso de todos los subsistemas de la institución y fortalecer el proceso de comunicación interna y externa. Así como también dinamizar gerencias institucionales, tales como: la Gestión de Información (GI), la Gestión Documental (GD) y la Gestión del Conocimiento (GC), entre otras (Rivero Amador, 2011).

Estas tres gerencias tienen una estrecha relación sistémica en el marco del desempeño de la propia organización como sistema abierto y complejo. Es reconocida la intermediación de la GI como integradora y desarrolladora de estos procesos organizacionales. Es, la gerencia de la información, la responsable de la interacción constante de los SI en los ambientes organizacionales y su total implicación con los objetivos institucionales (Mena, 2007; Ponjuán Dante, 2003). Desde esta perspectiva, la verdadera naturaleza del SI radica en su objetivo dentro de la institución, más que en su esencia (está centrado en conocer el para qué, más que en el cómo o el qué) (Andreu, Ricart y Valor, 1996; Piattini, Calvo Manzano, Cervera, y Fernández, 1996). A partir de estos cimientos, los SI se expresan en la literatura científica como sistemas integrados por subsistemas que responden a la satisfacción de necesidades de una institución, de un individuo o grupo de individuos. Son diseñados para responder a objetivos concretos, previamente estudiados, en el ambiente donde éstos se aplican. En su operación e implementación se debe comprobar el grado de eficiencia del mismo. Además, constituyen un conjunto de elementos o componentes relacionados con la información que interaccionan, para cumplir con un objetivo principal: facilitar y/o recuperar información de forma oportuna (Laudon, 2000; Ponjuán Dante, Mena, Villardefrancos y León, 2004).

En la praxis cotidiana de cualquier SI se producen los procesos de almacenamiento, identificación, transformación, organización, tratamiento y recuperación de la información. En cada uno de los pasos interviene la tecnología, que facilita el cumplimiento de los usos y funciones de la información (Moreiro, Sánchez y Morato, 2012). Como resultado se alcanzan cambios en el estado del conocimiento que poseen las personas, la solución de problemas informativos, o la toma de decisiones. Desde esta perspectiva, los SI, son esencialmente artefactos de conocimiento que capturan y representan este recurso en ciertos dominios (Barchini, Álvarez, Herrera, y Trejo, 2007). Este enfoque muestra la 
relación entre los conceptos información y conocimiento. La definición enunciada se refiere a los SI como herramientas intermediarias entre la diseminación de la información y su explotación de forma dinámica para crear conocimiento. Según el propio Nonaka, la información es un conjunto de materiales necesarios para iniciar y formalizar el conocimiento, y puede ser vista desde las perspectivas sintáctica y semántica (Nonaka, 1994):

- El aspecto sintáctico de la información: está basado en el análisis del volumen de información (esta medida es sin tener en cuenta su valor). El flujo de la información será más o menos representativo en función de cuán voluminosa sea la información. Este aspecto está más relacionado con las tecnologías informáticas a utilizar para lograr varios formatos en los que se guarden la información.

- El aspecto semántico de la información: es el más relevante de cara a la creación de conocimiento, puesto que desde este punto de vista se considera a la información en el contexto organizativo. De este modo se evita la tendencia generalizada de ver la información como un elemento a procesar a través de los SI, sin tener presente el objetivo por el cual se procesa y se disemina. El tratamiento de la información en el contexto de la institución es lo que permite la creación de conocimiento.

Mientras que los datos se encuentran en los registros sobre las transacciones y la información aparece en los mensajes, el conocimiento se encuentra en los individuos o grupos de conocedores $y$, a veces, en las rutinas organizativas. No obstante, su transmisión se produce a través de medios estructurados tales como libros, documentos, fotos, medios audiovisuales, etc. Todos constituyen formatos que pueden ser diseminados a través de los SI. De este modo, mediante esta interacción, se contribuye a que el conocimiento no sea solo transmitido mediante el contacto personal.

Después del estudio de la literatura científica se puede afirmar que los SI están constituidos por un conjunto formal de procesos que, operando sobre una colección de datos e información (estructurados según las necesidades de la institución), recopilan, elaboran y distribuyen la información (o parte de ella) necesaria para las operaciones, la toma de decisiones y la comunicación de los procesos que conforman la institución. En la propia esencia de estos procesos surge y se desarrolla el conocimiento organizacional, alimentando el conocimiento de los usuarios, que interactúan con estas plataformas o herramientas. Las instituciones modernas precisan de potentes SI diseñados a favor de la toma de decisiones, el proceso de comunicación y el desarrollo del conocimiento a nivel institucional.

En este escenario teórico-conceptual se desarrollan los Sistemas de Información Científica (SIC). Estos, desde la perspectiva teórica, abarcan máquinas y/o métodos organizados de recolección de datos, procesamiento, recuperación, transmisión y difusión de la información útil para los usuarios (Navarrete y Banqueri, 2008) y su contenido se corresponde a algún área de la ciencia (Cañas y Lorenzo, 2006). Los SIC tienen por objetivo fundamental, en el ámbito institucional, la estructuración de la información operativa y funcional que requieren las organizaciones para operar eficientemente y 
alcanzar resultados emprendedores. Estas plataformas desarrollan y apoyan a nivel macro las políticas científicas y tecnológicas que necesitan las organizaciones y sus procesos, con el objetivo de: definir y evaluar las estrategias seguidas en el desarrollo de las actividades de investigación, desarrollo e innovación; evaluar la producción y actividad científico/tecnológica de sus investigadores e instituciones; así como fomentar la colaboración, intercambio y transferencia de conocimientos (Armas, Díaz y Giraldes, 2008).

Este tipo de sistema puede ser orientado hacia la utilización de diversas fuentes de información para analizar el comportamiento de la ciencia a nivel institucional, regional o internacional. Un ejemplo representativo son los Sistemas de Información Curricular (sistemas que utilizanel curriculum vitae -CV- como fuente de información). La relevancia de estos sistemas radica en que los CV de los investigadores proporcionan información sobre publicaciones que generalmente no son recogidas por las bases de datos tradicionales. Estos documentos son los únicos que informan, con un importante nivel de detalle y riqueza analítica, acerca de las actividades académico-profesionales realizadas por los investigadores (incluyendo dónde y con quiénes trabajan), sus características sociodemográficas, sus trayectorias educativas, los productos realizados y otros aspectos específicos de sus trayectorias (como los patrones de colaboración científica, de movilidad geográfica $\mathrm{y} / \mathrm{o}$ institucional, los reconocimientos obtenidos, etc.) (Dietz, Chompalov, Bozeman, O'Neil y Park, 2000).

A pesar de que aún no es suficiente el nivel de normalización y estandarización de los campos de los CV, aspecto que se encuentra en plena realización por parte de varios especialistas, este documento se ha convertido en una fuente de información privilegiada para establecer patrones en la medición de la ciencia y la tecnología que puede ser complementada (e incluso potenciada) con otras fuentes de información como las encuestas, las bases de datos bibliográficas y de patentes disponibles. Esta filosofía de trabajo ha mostrado avances representativos en las materias métricas de la región iberoamericana, desde la integración de Sistemas de Información Curricular institucionales a sistemas regionales (Cañibano, Otamendi y Solís, 2010; Jaramillo, Lopera y Albán, 2008). Esta propuesta puede ser una herramienta favorable a la organización del conocimiento institucional, para su medición mediante el establecimiento de patrones teóricos-metodológicos que contribuyan a disminuir las barreras establecidas por las normalizaciones disciplinares y su contraste con la interdisciplinariedad de los resultados científicos. De igual forma se convierten en herramientas favorecedoras de la GC, ya sea a nivel institucional o regional.

La medición de la ciencia y la tecnología en la región latinoamericana precisa de instrumentos, metodologías y herramientas novedosas para divulgar, incrementar y evaluar los resultados de la ciencia y la tecnología. Un imperativo en la actualidad es el perfeccionamiento de normativas, políticas regionales $e$ indicadores regionales normalizados, ajustados a las nuevas potencialidades y características específicas de nuestra región. Por otro lado, es igual de necesario el diseño e implementación de SIC regionales, enfocados a las características de nuestras instituciones, en plena armonía con los patrones internacionales y en completa interacción con indicadores pertinentes que contribuyan a la toma de decisiones y medición del conocimiento institucional y regional. 
América Latina se ha caracterizado por una amplia influencia del sector universitario como protagonista en la producción y diseminación del conocimiento de la región. Por consiguiente, la construcción de indicadores métricos con fines evaluativos, la aplicación de metodologías y sistemas de políticas para gestionar la ciencia y la tecnología, así como la implementación de SIC en estas instituciones, se convierten en factores estratégicos a tener en cuenta para impulsar la producción científica del resto de las instituciones del Sistema de Ciencia e Innovación Tecnológica (SCIT) y el desarrollo de políticas nacionales desde los organismos rectores de la política científica de los países (Arencibia, 2012; Barandiarán y D'Onofrio, 2013).

En este escenario, Cuba ha venido desarrollando varias acciones para fortalecer la medición de los resultados de la ciencia y la tecnología en los diferentes niveles. Estas acciones siguen siendo incipientes respecto a la necesidad imperiosa de sistematizar prácticas que favorezcan la implementación de metodologías de medición desde la rigurosidad de los estudios cuantitativos de la producción científica nacional. El desafío actual de los estudios cuantitativos de la ciencia es rebasar la mera aproximación cuantitativa e incidir en los procesos de toma de decisiones estratégicas encaminados a impulsar, consolidar o perfeccionar la evaluación de la actividad científica realizada en el país (Arencibia, 2012).

La presente investigación se concentra en esta problemática y se nutre de patrones teóricos y metodológicos de la literatura científica con el objetivo de desarrollar un instrumento de medición de la ciencia y la tecnología en instituciones universitarias, utilizando como herramienta el Sistema CV-UPR. Para la validación de la propuesta se toma como caso de estudio la Universidad de Pinar del Río.

\section{Materiales y métodos}

La investigación se basa en la revisión bibliográfica y el análisis documental de importantes referentes metodológicos y conceptuales. Se parte de premisas sustentadas en los patrones metodológicos establecidos en los manuales de ciencia y tecnología a nivel internacional o regional, de obligada consulta para establecer este tipo de indicador de medición, desde una perspectiva normalizada. Los principales manuales consultados fueron: Manual de Frascaty (OCDE, 2002), el Manual de Canberra (OCDE, 1995), el Manual de Lisboa (RICYT, 2009) y el Manual de Santiago (RICYT, 2007). Es de una importancia muy puntual el Manual de Buenos Aires, que se encuentra en realización. Este manual se concentra en la construcción de indicadores de trayectorias de investigadores científicos y tecnológicos, tomando el curriculum vitae (CV) de los investigadores como fuente de información (D’Onofrio, Solís, Tignino y Cabrera, 2010). Estos manuales constituyen premisas para la adecuación de indicadores regionales de ciencia y tecnología a la realidad institucional desde la propia contextualización en nuestra región. En este sentido, se enmarca el comportamiento del investigador en relación a la institución a la que pertenece para medir el desempeño en el proceso de ciencia y tecnología con la influencia de estos dos factores (investigador-institución) (D’Onofrio, Solís, Tanino y Cabrera, 2010).

Se toma como caso de estudio el análisis del proceso de ciencia y tecnología en la 
Universidad de Pinar del Río (UPR), y para validar el instrumento de medición propuesto se toma como espacio temporal un total de 5 años, desde el 2010 al 2014. Como parte del análisis documental fueron consultados los informes de la institución objeto de estudio en el proceso de ciencia y tecnología, así como sus principales documentos metodológicos y estratégicos de los años 2010-2014. Conjuntamente, se utilizó el método empírico de la observación y las entrevistas y encuestas, como técnicas empíricas para obtener información de los procesos de la ciencia y la tecnología en la institución. Estas técnicas empíricas que se aplicaron, favorecen la recolección de la información necesaria para diagnosticar el comportamiento de la actividad investigativa de la institución, referente a los resultados científicos de los investigadores.

En el caso de los cuestionarios aplicados se utilizan dos formas distintas para seleccionar los encuestados. La población objeto de estudio, para el primer cuestionario, está conformada por los investigadores pertenecientes a la universidad que ostentan el grado científico de Doctor en Ciencias. La población analizada está conformada por un total de 118 doctores en ciencia de determinada especialidad que se encuentran titulados o defendidos hasta el año 2013. Para el cálculo de la muestra se utilizó el Muestreo Irrestricto Aleatorio (MIA), donde se utilizó la fórmula de Calero (1978). Esta fórmula estadística expresa que el tamaño necesario de la muestra, para la población identificada en el estudio de esta investigación, es de 90 encuestados. Esta muestra fue dividida por estratos, los cuales fueron declarados en función de las especialidades de los doctorados (según establecen los informes de la institución).

La segunda encuesta fue aplicada a los coordinadores de proyectos de investigación, la población objeto de estudio está conformada por los investigadores pertenecientes a la universidad que tienen la responsabilidad de coordinar proyectos de investigación. Para este estudio se toma el listado de proyectos de investigación en el período 2011-2013. De este modo se tiene en cuenta proyectos que están vigentes o en ejecución y pertenecen a la sede central de la UPR. Este período de tiempo fue el acordado para hacer este tipo de estudio empírico dentro del cronograma de la investigación. Al seguir los criterios explicados con anterioridad, se declara una población de 33 investigadores, que son coordinadores de proyectos, en el período 2011-2013. Se aplica el cuestionario a la totalidad de la población identificada.

En el procesamiento de los datos se utilizó el software Statistical Package For Social Science (SPSS, versión 11.5, 2004). Además, se utiliza como herramienta el Sistema de Gestión de Información y Conocimiento Institucional de la Universidad de Pinar del Río (CV-UPR), desarrollado por el grupo proGINTEC de esa institución académica. Esta plataforma constituye la principal fuente de información para diseñar y validar el instrumento de medición de la ciencia y la tecnología que se propone por la investigación, tomando como caso de estudio la referida institución. El sistema se encuentra implementado hace varios años en la referida universidad. Para la validación de los indicadores diseñados se utilizaron los CV de los investigadores con un rango de tiempo de 5 años desde el 2010 hasta el 2014. 


\section{Resultados}

El instrumento propuesto en esta investigación utiliza como principal fuente de información la estructura curricular que presenta el Sistema CV-UPR, de la Universidad de Pinar del Río, ya abordado con anterioridad. Esta plataforma establece una estructura curricular ajustada a las características de la institución. Cada investigador de la institución actualiza su CV de manera periódica, mediante su usuario individual, y es el responsable de suministrar los datos que se utilizan para elaborar los indicadores de medición. En este sentido, para gestionar los CV de los investigadores de la institución, el Sistema CV-UPR parte de premisas estructurales establecidas en el curriculum vitae para Latinoamérica y el Caribe (CVLAC), también nombrado Curriculum Lattes. Esta plataforma curricular es muy usada en nuestra región, por lo que su utilización como premisa estructural favorece la normalización de los campos del CV en su utilización para generar indicadores de medición más normalizados (Armas, Díaz y Giraldes, 2008). Para la elaboración del instrumento de medición propuesto se utilizan como fuente de información los siguientes ítems del CV de los investigadores:

- Datos Personales.

- Experiencia Laboral.

- Historial Académico.

- Experiencia Docente.

- Experiencia Científica Tecnológica.

- Estancias en Instituciones nacionales y Extranjeras.

- Producción Científica y Técnica.

- Participación en Eventos.

- Premios.

- Reconocimientos.

El instrumento de medición está compuesto por 6 variables con objetivos comunes (Figura 1) en los resultados cuantitativos del proceso de ciencia y tecnología a nivel institucional. Las variables agrupan a un total de 70 indicadores estructurados en patrones comunes de medición. Esta estructuración permitió establecer un análisis específico de determinadas actividades relacionadas con la gestión de la ciencia y la tecnología y a la vez establecer contrastes en los resultados de los indicadores.

Los indicadores diseñados, a pesar de ser agrupados en las variables, pueden ser contrastados entre sí. Esta delimitación por objetivos más específicos responde a características específicas de cada dato proveniente del CV del investigador $y$, una vez contextualizado y procesado por el Sistema CV_UPR, puede ser consultado en los indicadores calculados mediante las bondades gráficas de esta plataforma. A continuación se explican los detalles de cada variable establecida (Figura 1): 
Figura 1. Composición de las variables del instrumento de medición de la ciencia y la tecnología a nivel institucional

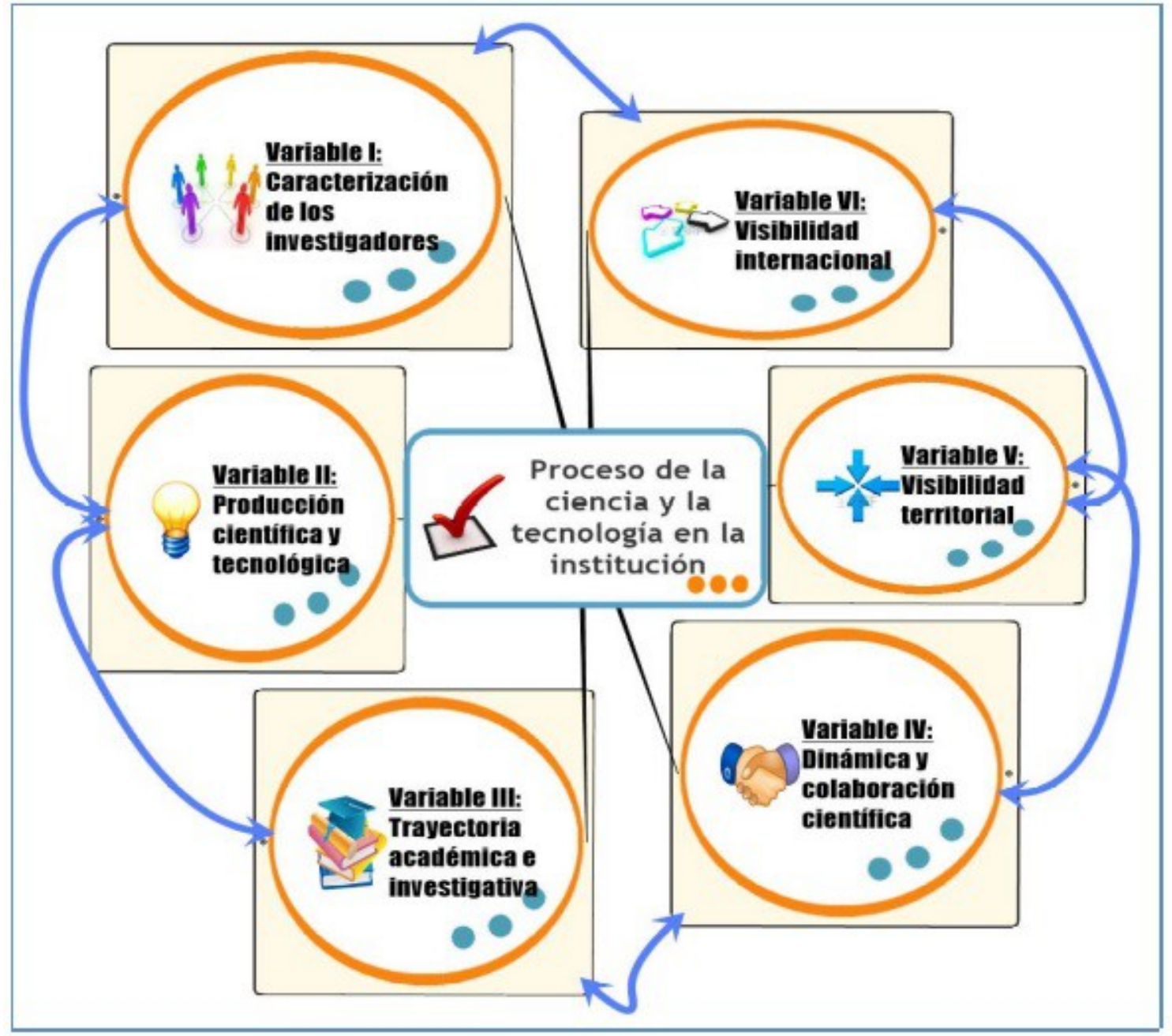

Fuente: elaboración propia.

- Variable I. Caracterización de los investigadores: es el punto de partida de la medición, está compuesta por 15 indicadores. Es retomada cuando se analizan los resultados de las restantes variables para comparar resultados específicos de los investigadores con los de la institución. Estos aspectos que caracterizan a los investigadores y su comportamiento, en un espacio de tiempo determinado, ayudan a entender las tendencias favorables o desfavorables de los resultados científicos de la institución. Además, es una de las formas de comparar los resultados investigativos institucionales, que se obtienen en un período de tiempo específico, respecto a la potencialidad formativa y las capacidades que poseen los investigadores que componen la institución.

- Variable II. Producción científica y tecnológica: aglutina aspectos propios de la producción científica y tecnológica de la institución que se analiza. En esta variable, un total de 14 indicadores estructuran la producción científica de la institución y hacen énfasis en las características de las publicaciones científicas 
como uno de los aspectos de gran relevancia de esta producción. Además, se establecen parámetros que identifican determinados resultados científicos provenientes de proyectos de investigación.

- Variable III. Trayectoria académica-investigativa: complementa el análisis de la producción científica y tecnológica de la institución (aspecto que se estudia en la Variable II) y distingue la repercusión que tiene la investigación científica en el desarrollo de la actividad académica de la institución. Esta característica, distintiva de instituciones universitarias, precisa la obtención de información relacionada con los procesos investigativos y académicos para valorar el desempeño institucional balanceado en estos dos aspectos tan relevantes para la excelencia de la institución. En este sentido, se proyecta el objetivo de la interpretación de la Variable III, estructurada en 12 indicadores.

- Variable IV: Dinámica y colaboración científica: posibilita el estudio de la dinámica y colaboración científica que se desarrolla en la obtención de los resultados de la ciencia y la tecnología de la institución. Este aspecto expresa el nivel de socialización y divulgación del conocimiento científico institucional. Esta variable se estructura en 11 indicadores que estiman las relaciones colaborativas con otras instituciones o investigadores para obtener resultados científicos en conjunto.

- Variable V. Visibilidad territorial: resalta el papel estratégico que distingue la influencia de estas instituciones en el desarrollo del territorio o región en donde se desempeñan. Esta misión de la universidad en su extensión hacia la comunidad, justifica la necesidad de contar con patrones de medición que resalten los resultados científicos y su visibilidad a nivel territorial. Con este objetivo se estructura esta variable, y está compuesta por un total de 9 indicadores.

- Variable VI. Visibilidad internacional: efectúa una aproximación a la medición de la internacionalización de la ciencia a nivel institucional. La variable VI se estructura en 9 indicadores que expresan valores cuantitativos que permiten valorar la visibilidad internacional que posee la institución, en un período determinado y como consecuencia del desempeño de sus investigadores y las actividades de colaboración internacional en las que participan éstos.

Para realizar el diseño de los indicadores y su descripción detallada de cálculo fueron consultadas varias experiencias, desde la perspectiva de la cienciometría y la bibliometría. De igual modo se consultaron los enfoques relacionados con el diseño de indicadores de gestión, indicadores de desempeño, indicadores de calidad de los procesos de gestión e indicadores estratégicos, que se pueden consultar en la literatura especializada de las ciencias empresariales (AENOR, 2003; Catedral, 2012; CONEVAL, 2010; Greco, 2012; Reginaldo y González, 2012). Como resultado de este análisis se obtiene un sistema de indicadores para ser utilizados en la aplicación del instrumento de medición propuesto por la investigación. 


\section{Análisis y discusión}

En el período 2010-2014 la Universidad de Pinar del Río desempeñó sus funciones académicas e investigativas con un total de 515 investigadores, identificados en el Sistema CV-UPR como profesores-investigadores que desempeñaron cargos en áreas docentes o investigativas. La variable I permite establecer una caracterización de los investigadores que se desempeñaron en el periodo de evaluación. A continuación se plantea un compendio de los principales resultados luego de la aplicación del instrumento mediante el análisis de las variables de medición definidas.

La variable I permite realizar una caracterización de los investigadores; se puede identificar que de un total de 515 investigadores, 273 representan al sexo masculino y 242 al sexo femenino, con un 54\% y 46\%, respectivamente. Se observa una amplia incorporación de la mujer y un equilibro de género en la institución. En el período que se analiza, la institución cuenta con un $40 \%$ de sus investigadores con más de 10 años de antigüedad $y$, aunque no es una cifra alta, se puede valorar este criterio como positivo y favorecedor del incremento del conocimiento institucional desde la experiencia de sus investigadores. Además existe un total de 15 investigadores que obtienen el grado científico de doctor en este periodo de tiempo, representando un $3 \%$ de la totalidad de doctores de la institución. La institución tiene como política la inclusión de investigadores jóvenes en la formación doctoral; en este sentido se muestra un $75 \%$ de aspirantes al grado científico de doctor, con edades menores a 35 años de edad. En cuanto a las características de la formación de los investigadores, se puede anunciar que en este período la institución muestra un total de 358 investigadores con grado científico, representando el $70 \%$ del total, con perspectivas de incrementar esta cifra en años venideros. Con este fin, se encuentran 241 investigadores en proceso de formación, lo cual representa un $46 \%$ del total de investigadores de la institución.

La participación de investigadores en proyectos de investigación alcanza el $50 \%$ del total de investigadores, y de ellos el $38 \%$ se desempeñaron como coordinadores de proyectos. Del total de los investigadores con grado científico, el 69\% imparte actividades de postgrado. Además, el $15 \%$ del total de investigadores con grado científico tiene responsabilidades de dirección, relacionadas con el proceso de ciencia y tecnología. Estos indicadores muestran una implicación favorable de los investigadores en actividades que apoyan y desarrollan la ciencia, la tecnología y el conocimiento institucional.

Desde otra perspectiva, del total de investigadores, el $72 \%$ realizaron publicaciones científicas; de éstos, un $19 \%$ obtienen resultados que interaccionan con varias áreas del conocimiento. Estos investigadores están formados en tres áreas del conocimiento fundamentales: Ciencias forestales, Ciencias económicas y Pedagogía. Este indicador muestra síntomas para la identificación de fortalezas de la institución en estas áreas que ya se han distinguido en los análisis de las técnicas empíricas aplicadas.

Para realizar un estudio más profundo deben ser analizados los siguientes indicadores: el Índice Promedio de Actuación Investigativa y el Índice Promedio de Actuación Académica. Estos dos indicadores realizan un promedio entre las cantidades de acciones realizadas por los investigadores de la institución en la esfera investigativa y en la académica. Ambos índices se analizan en conjunto para valorar el peso de la actuación de la institución en el período que se evalúa. En la Figura 2 se representan los valores de los índices promedio 
de actuación investigativa y académica en los 5 años del período de análisis. En este período se puede observar que el mayor peso recae en la actividad académica, la cual tuvo un incremento favorecido por la actividad de posgrado, pues la docencia que los investigadores impartieron en el pregrado se mantiene con cantidades relativamente constantes.

Figura 2. Índice promedio de actuación investigativa en relación con el Índice promedio de actuación académica

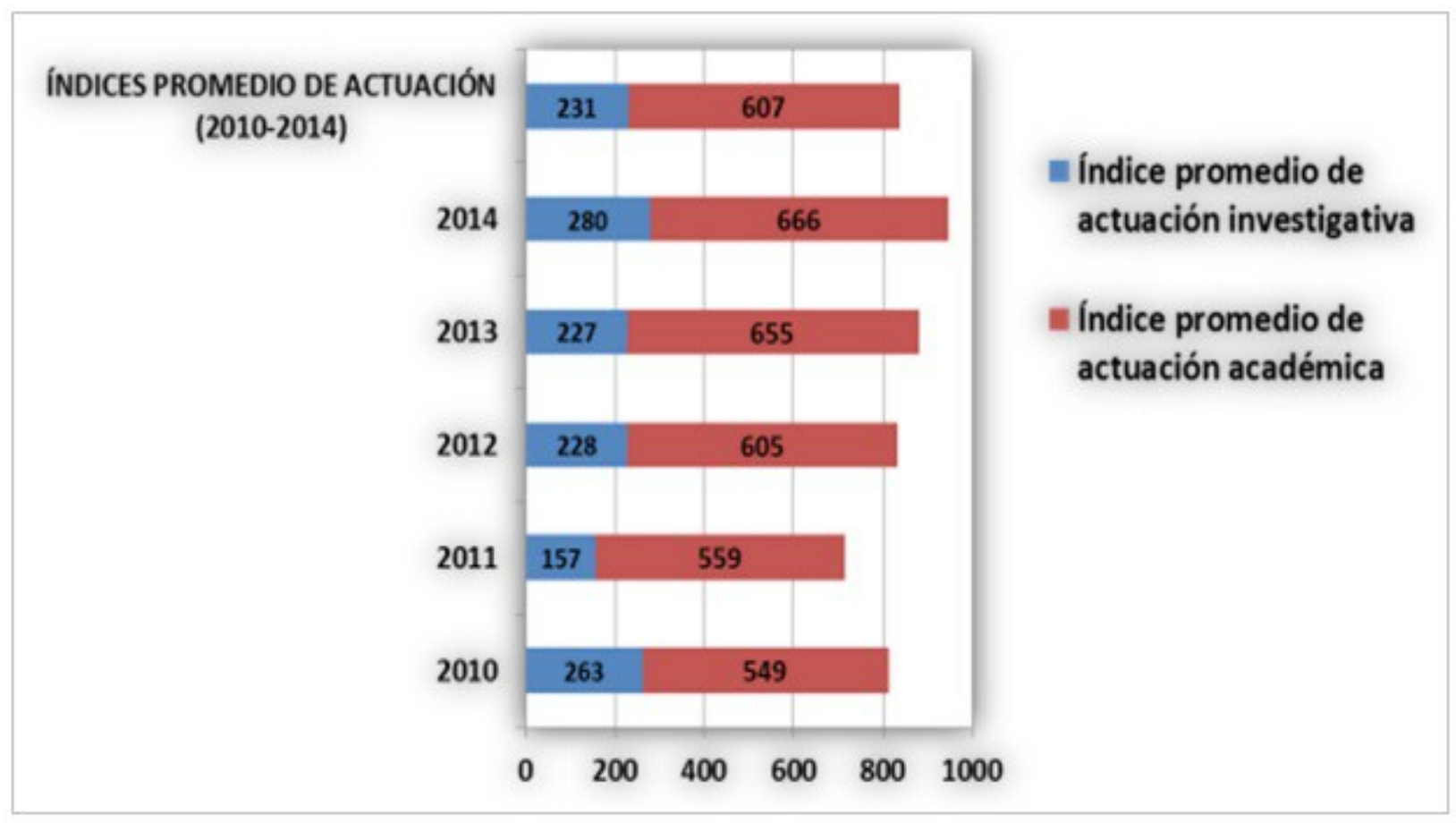

Fuente: elaboración propia.

Existe una relación desequilibrada entre los dos índices, lo cual muestra que el peso de la actividad de la institución descansa en la academia, mientras que los resultados científicos han tenido un comportamiento inestable, siendo el año 2011 el de menor cantidad de producción científica. Esta misma inestabilidad al aumento y disminución se ha observado en la producción científica del período. Estos indicadores demuestran que los investigadores obtienen más cantidad de resultados académicos respecto a los científicos. Para visualizar un resultado positivo en este tipo de indicador, en caso de existir desequilibrio entre los dos índices, el peso mayor lo debería tener el índice de actuación investigativa, pues las actividades académicas deben tributar a la actividad de proyectos de investigación, a la publicación de resultados científicos provenientes de las tesis de maestría y doctorado. Las cantidades de acciones académicas, en un período de cinco años, deben ser valores que tiendan a ser poco cambiantes o estables en el aumento cuantitativo. El comportamiento favorable debería ser hacia el incremento del índice promedio de actuación investigativa, y el factor más determinante en el aumento debería ser la publicación científica de los investigadores, aunque la actividad de proyectos de investigación tiene un peso importante. El aumento del índice promedio de actuación académica evidencia una mayor cantidad de tesis de postgrado defendidas y asesoradas, pero escasa publicación de resultados y poca visibilidad del conocimiento resultante. 
La variable II parte del cálculo de la producción científica y tecnológica de la institución en el período seleccionado. La producción científica de este período se comportó de manera inestable, con ascensos y descensos en cada año y el año menor producción fue el 2011. La mayor representatividad de esta producción se refleja en los resultados de proyectos de investigación, la publicación de libros, capítulos de libros y monografías y la publicación de artículos en revistas, sumando éstos un total de 1155 en los cinco años. Estos criterios de la producción científica tuvieron un comportamiento similar a lo largo de este período. Desde otra perspectiva, la producción tecnológica tiene un comportamiento descendente, existe mayor incidencia en los registros informáticos y la cantidad de patentes solicitadas y concedidas es mínima, ya que solo fueron solicitadas 3 patentes y en este período fueron concedidas. Es evidente que la institución tiene mayor repercusión en los resultados científicos.

En la publicación científica de este período se visualizan determinadas áreas del conocimiento con mayor representatividad. La Figura 3 muestra las publicaciones científicas de la institución, por áreas del conocimiento científico. Prevalecen las publicaciones en las Ciencias forestales, las Ciencias económicas, las Ciencias agrícolas, la Pedagogía y las Ciencias técnicas; el resto de las áreas poseen cantidades menores. Las áreas del conocimiento más representativas manifiestan fortalecimiento en la socialización del conocimiento y una mayor inclinación de sus investigadores a publicar y visibilizar sus resultados científicos.

Figura 3. Publicaciones científicas por área del conocimiento

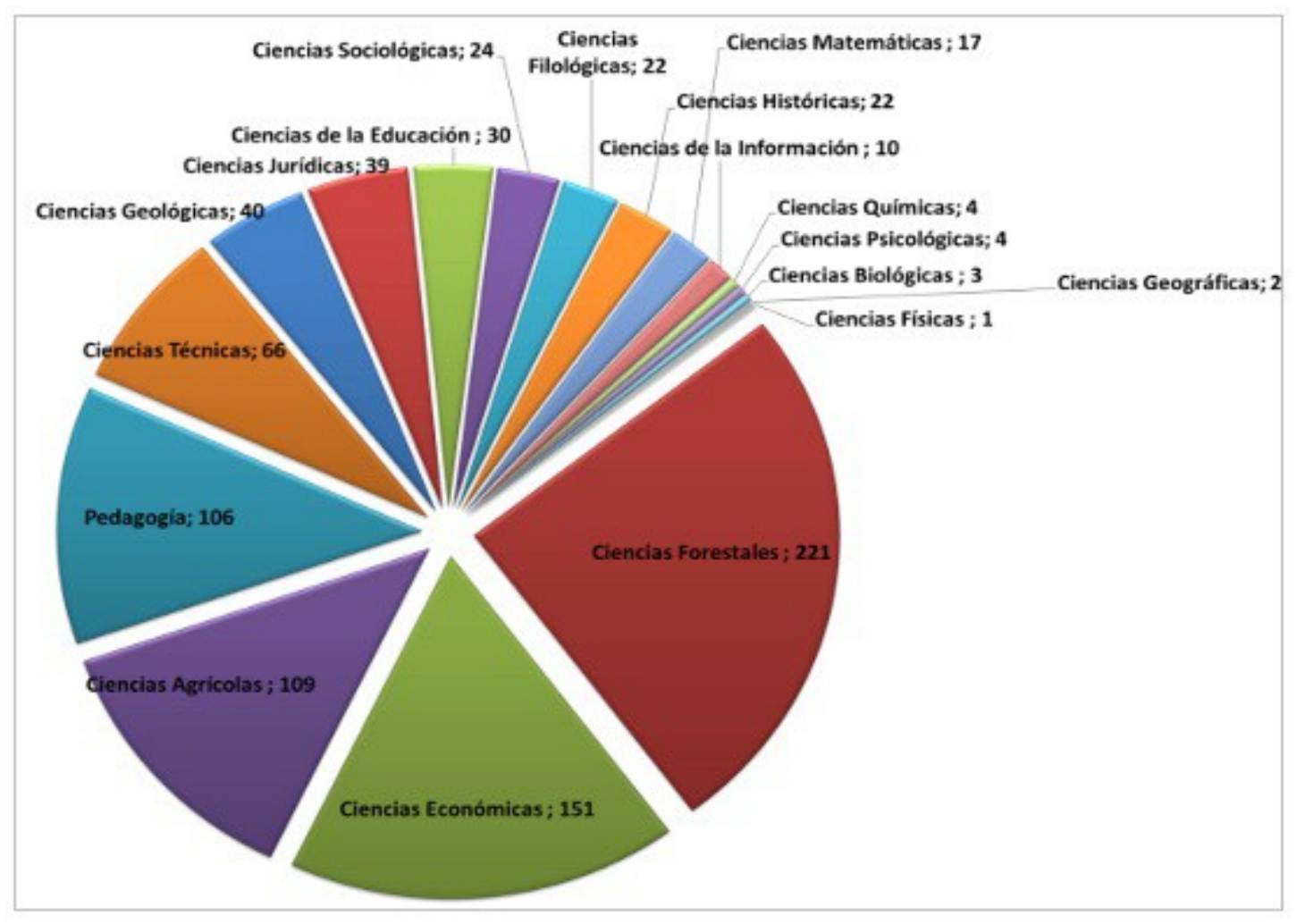

Fuente: elaboración propia.

Otro indicador en la publicación científica muestra que la productividad por investigador, en la publicación de artículos en revistas, se comporta a un promedio de 1.88 artículos en 
cinco años. Este valor es poco significativo y es el resultado de que solo el $72 \%$ de los investigadores realizaron publicaciones en el espacio temporal que se estudia, y de ellos el $20 \%$ tienen una mayor productividad ya que han publicado más de 6 artículos. Este comportamiento en la publicación científica limita la socialización del conocimiento de la institución. En este sentido, las revistas científicas son uno de los principales canales de comunicación y difusión de los resultados de investigación y de institucionalización social de la ciencia en la mayoría de los campos del conocimiento $y$, aunque no todas tienen el mismo prestigio y grado de influencia en la comunidad científica, pues su reconocimiento depende en gran medida de su calidad y su visibilidad, es muy necesario que se logren determinados volúmenes de publicaciones (Miguel, 2011). En las entrevistas realizadas a especialistas de la gestión de la ciencia y la tecnología de la institución se corrobora el bajo índice de publicación en revistas científicas influenciado por la poca motivación de los investigadores, y la necesidad de incrementar el asesoramiento y capacitación para desarrollar esta actividad como fase del proceso de investigación. Además, los entrevistados reconocen que es escaso el trabajo en equipos de investigadores materializado en la actividad de proyectos de investigación y la necesidad de lograr la publicación científica como un objetivo en interacción conjunta.

Otro aspecto a medir en esta variable es la procedencia de la publicación de artículos científicos. El total de publicaciones en el período se desglosa en cuatro tipos de procedencia de los resultados que se publican: asesorías de tesis, investigación de doctorado, resultados de proyectos de investigación, investigaciones de maestría o especialidad, así como otras procedencias. Un aspecto preocupante es que los investigadores identifican en un $45 \%$ que sus publicaciones provienen de otras procedencias, lo cual muestra síntomas de independencia en la publicación científica, es decir gran cantidad de publicaciones con autoría única, además de la existencia de investigadores que no se integran a la actividad de proyectos y no publican totalmente los resultados que se obtienen en sus actividades académicas. Un resultado similar se obtiene al valorar el nivel de impacto de las revistas científicas donde se publica. Un $70 \%$ de las publicaciones se encuentran en revistas indizadas en bases de datos de reconocimiento latinoamericano, y solo el $12 \%$ de las publicaciones se realizan en revistas de corriente principal, con visibilidad internacional. De este modo, se observa un menor nivel de calidad y visibilidad en el total de publicaciones de este período.

Si se pone el énfasis en un grupo de indicadores que caractericen la autoría de las publicaciones en revistas científicas, se puede resaltar que el nivel de independencia en la publicación de artículos se comportó en un 0.27 , lo que significa que el $27 \%$ de los investigadores publicaron artículos como autor único. Esto contrasta con la tendencia a incrementar la autoría múltiple y el desarrollo de resultados en coautoría para desarrollar la dinámica de trabajo colaborativo en la creación del conocimiento. Por su parte, la interdependencia externa en la publicación se identifica con un 0.45 , lo que significa que el $45 \%$ de los artículos publicados representan la coautoría con investigadores de otras instituciones, mientras que el protagonismo en la publicación de artículos se muestra en un 0.88 , lo que expresa que el $88 \%$ de los artículos corresponden a la autoría principal de los investigadores de la institución. En este sentido se aprecia un comportamiento favorable hacia la autoría de los investigadores de la institución, pero es de vital 
importancia el incremento de la publicación de artículos en colaboración con autores internos y externos a la institución para lograr incentivar el intercambio y favorecer el desarrollo del conocimiento institucional.

En el caso de los proyectos de investigación, se valoran tres indicadores fundamentales: los proyectos en ejecución en el período, los proyectos de investigación con propiedad intelectual y los proyectos de investigación con resultados en varias áreas del conocimiento. En el período que se analiza, existen 282 proyectos de investigación en ejecución en total, de los cuales un $15 \%$ posee propiedad intelectual, mayormente en registros de productos informáticos. De esta totalidad de proyectos en ejecución, un $18 \%$ interacciona con varias áreas del conocimiento en la obtención de resultados científicos y en la interacción con varios especialistas. Los resultados científicos de estos proyectos se relacionan con varias áreas del conocimiento. Estas interacciones con varias áreas del conocimiento en el desarrollo de un proyecto de investigación con un objetivo común se evidencian en relaciones con varias instituciones y diversos especialistas. Este comportamiento también fue evidenciado en las encuestas realizadas, en las que el $80 \%$ de los coordinadores encuestados declararon que sus proyectos obtienen resultados científicos relacionados con varias áreas de la ciencia.

El análisis de la variable III permite identificar que en el periodo de medición existe mayor relevancia en la esfera académica en las siguientes áreas de la ciencia: las Ciencias agrícolas, las Ciencias Forestales, las Ciencias económicas y las Ciencias técnicas, ya sea en programas de pregrado o postgrado. Además del total de programas de postgrado impartidos, un $21 \%$ tienen mayor permanencia, con más de 5 ediciones. En este período, se han fortalecido los programas de pregrado y postgrado con la incorporación de 136 doctores en los programas de pregrado y 123 a programas de postgrado para un $90 \%$ de implicación de investigadores con grado científico de doctor en las actividades académicas. El nivel de asesorías tutelares en el postgrado se comportó en un 4.17, y además existen un $26 \%$ de doctores miembros de tribunales nacionales de doctorado.

En este período se defienden un total de 192 tesis de postgrado en diversas áreas del conocimiento, de las que destacan las Ciencias forestales, las Ciencias agrícolas, las Ciencias económicas y la Pedagogía. Se puede apreciar que, en este período, los resultados de las investigaciones que provienen de la actividad académica se asocian a proyectos de investigación, ya sean de la propia institución o de otras, y así lo expresa el $98 \%$ de las tesis de postgrado defendidas en estos cinco años. Además, el $88 \%$ de los proyectos de investigación que se encuentran en ejecución incorporan estudiantes de pregrado en el cumplimiento de los objetivos investigativos. Esta actividad necesita de un seguimiento constante para lograr una mayor incorporación de investigadores en la creación de conocimiento científico en interacción grupal, como así también más socialización y aprendizaje. Para lograr este propósito se necesita una mayor implicación de los investigadores en la actividad de proyectos.

La actividad académica de la institución necesita un incremento de las publicaciones científicas asociadas a estos resultados. A pesar de que el $100 \%$ de los departamentos investigativos de la institución están asociados a actividades académicas, y de igual modo los departamentos docentes obtienen resultados investigativos, aún no se expresa en la 
producción científica una armonía respecto a la actividad académica desempeñada. Por ejemplo, solo el $28 \%$ de los resultados académicos fueron publicados, lo cual expresa que las tesis de postgrado no han sido publicadas de acuerdo con los requerimientos de este tipo de formación. Sin embargo, existe tendencia a utilizar publicaciones científicas para la actividad académica, tal es el caso del $43 \%$ de los libros publicados que han sido destinados a la actividad académica de pregrado y postgrado.

El estudio de la variable IV permite afirmar que la dinámica y colaboración científica de la institución, en el período de tiempo seleccionado para el análisis, se refleja más fortalecida hacia el territorio y la nación. Este comportamiento se expresa mediante la publicación científica de manera conjunta con especialistas de otras instituciones, los convenios de colaboración con otras instituciones del territorio y las acciones conjuntas entre especialistas de otras Instituciones para la formación académica, entre otros aspectos cuantificados en el sistema de indicadores. Este comportamiento colaborativo fomenta el desarrollo endógeno de la producción científica de la institución, al mismo tiempo que enriquece determinadas variables exógenas que determinan el desarrollo del territorio.

El proceso de publicación, ya caracterizado en la variable II, presenta una dinámica poco favorable a la colaboración con otras instituciones. Por ejemplo, del total de publicaciones científicas en colaboración con otras instituciones solo el $5 \%$ tiene un carácter persistente, y el $3 \%$ de los artículos tienen autores de varias instituciones. Esta característica muestra que los investigadores no están publicando en interacción armónica con los colaboradores externos de proyectos de investigación o con cotutores de actividades de postgrado. En este sentido, la encuesta aplicada a los doctores evidencia que el 58\% de los encuestados publicaron sus resultados científicos en revistas nacionales, y que un $26 \%$ publicó como autor único.

Desde el punto de vista de la interacción de las áreas del conocimiento se observa mayor intercambio en la publicación científica con las mismas áreas de conocimiento. En este sentido, el $84 \%$ de las publicaciones científicas se realizan en colaboración con autores de una misma área del conocimiento, y el 14\% de las publicaciones pertenecen a autores de distintas áreas del conocimiento. Las áreas del conocimiento de mayor interacción coinciden con las ya abordadas de mayor fortaleza en la institución. La colaboración al interior de la actividad académica muestra rasgos muy favorables ya que el $46 \%$ de las tesis defendidas tienen asesoría de investigadores de otras instituciones, y el 39\% de los investigadores realizan asesorías en la formación de postgrado de otras instituciones. Estos aspectos deben lograr mayores incrementos para favorecer el desarrollo del conocimiento institucional.

Estos factores analizados han influido en que la institución adquiriera mayor visibilidad territorial y nacional, que favorece el impacto del conocimiento científico resultante del proceso de la ciencia y la tecnología a nivel institucional. No obstante, la producción tecnológica del dominio científico de la institución no refleja mayor apropiación y trasferencia para la generación de productos y servicios al interior del territorio. Se produce conocimiento con posibilidades de apropiación pero no se realizan con amplia representatividad los registros de patentes, ni de otro tipo. Estos aspectos siguen siendo asignaturas pendientes en la preservación de la producción científica institucional, y para 
fomentar estrategias puntuales de cooperación con empresas e instituciones territoriales que fomenten las capacidades de adsorción del conocimiento resultante del proceso investigativo en conjunto, que se materializa en proyectos de $I+D$, en colaboración universidad-empresa.

Las dos últimas variables indagan sobre la externalización de los procesos de ciencia y tecnología a nivel regional e internacional. Es tradicional la influencia y protagonismo de las instituciones universitarias como actores clave en el tejido social y su desempeño en actividades de formación y docencia, investigación y vinculación con el entorno socioeconómico que la rodea (D'Este, Castro y Molas, 2009). Aunque, de igual modo, la internacionalización de la ciencia se percibe en la actualidad como una condición necesaria para el desarrollo de la práctica científica en un mundo crecientemente interrelacionado. Además, constituye el camino más efectivo para lograr la mejora de la calidad de las actividades científicas y tecnológicas, la formación de recursos humanos altamente competentes, la socialización de la información y el conocimiento, así como para lograr estrategias permanentes en la cooperación con el objetivo de desarrollar el conocimiento científico, tal como se plantea en el Manual de Santiago (RICYT, 2007; Sebastián, 2011).

Desde la perspectiva territorial se manifiesta la relevancia de premios y distinciones a nivel territorial y nacional, estos premios son el reconocimiento de la actividad investigativa de la institución o de varios investigadores. Del total de premios y distinciones, el $93 \%$ pertenece a premios y distinciones territoriales, es decir en la provincia de Pinar del Río. Además, el $7 \%$ de los premios son de impacto nacional. De este modo, se observa un protagonismo de la institución en el territorio y la fortaleza de sus resultados científicos en relación con las problemáticas específicas del territorio. Por su parte, la actividad de proyectos de investigación tiene su manifestación directa hacia las problemáticas del territorio, así lo muestra el $60 \%$ de los proyectos de investigación que se enfocan en prioridades estratégicas del territorio, y el $34 \%$ de los proyectos que se relacionan con instituciones de gran impacto en el desarrollo económico y social del territorio. Otro aspecto a destacar es el incremento de la impartición de cursos y entrenamientos ajustados al estudio de necesidades de instituciones territoriales, en estrecha interacción con el desarrollo económico y social. En el período que se analiza fueron impartidos un total de 932 cursos y entrenamientos y 230 servicios de consultoría científica y tecnológica. Además, un total de 54 instituciones del territorio y de la nación han estado implicadas en la actividad académica e investigativa.

Las publicaciones científicas de la institución se proyectan hacia revistas nacionales, unas de mayor impacto y otras de relevancia nacional. La preeminencia de la publicación de los resultados científicos se orienta hacia este tipo de revista para lograr un alcance territorial y nacional ajustado al tipo de resultado muy orientado a características específicas.

Por su parte la internacionalización tiene un carácter más discreto, en primera instancia. Se evidencia un total de 5 premios internacionales en este período y la participación de la institución en 24 proyectos internacionales. Además, el $20 \%$ de los programas de posgrado aprobados han sido impartidos en el extranjero y 35 actividades de asesoría y consultoría académica. Otro aspecto relevante es que el $26 \%$ de las tesis de pregrado y posgrado asesoradas pertenecen a autores extranjeros. 
En cuanto a la visibilidad de los resultados científicos en la esfera internacional, existen aspectos que deben ser atendidos con la intención de incrementar las publicaciones científicas con impacto internacional. Al respecto, el $19 \%$ de las publicaciones científicas tienen impacto internacional, el $16 \%$ de las publicaciones son con autores de otros países. La mayor visibilidad internacional de los resultados científicos de la Universidad de Pinar del Río se encuentra en revistas referenciadas principalmente en Latindex, AGRIS y Forestry Abstracts, el $70 \%$ de los artículos publicados han sido en revistas que pertenecen a estas bases de datos. Existe otro núcleo de publicaciones visibles, aunque en menor medida (12\%), en ISIS y Scopus. Un aspecto favorable a la visibilidad de resultados científicos es la impartición de 96 conferencias en congresos internacionales, y la obtención de 48 resultados de investigaciones de posgrado con visibilidad en instituciones internacionales, producto de la adquisición de becas de investigación y la discusión de resultados investigativos en relación con instituciones internacionales.

El análisis de los resultados que se derivan, tras la aplicación del procedimiento de medición, permite afirmar que en estos cinco años el conocimiento institucional ha sido diseminado y socializado mediante la publicación de resultados científicos con mayor visibilidad en la región latinoamericana y en el marco nacional. Aún es incipiente la publicación en revistas de la corriente principal respecto a la cantidad de investigadores que se desempeñan en la institución, y específicamente referido a la cantidad de investigadores con grado científico y experiencia científica y académica.

Las áreas del conocimiento más fortalecidas en la publicación de resultados científicos son las Ciencias forestales, las Ciencias económicas, las Ciencias agrícolas, la Pedagogía y las Ciencias técnicas, en orden de productividad en la publicación. En estas áreas del conocimiento se manifiestan la mayor cantidad de interacciones en el proceso de investigación científica. En este sentido, se identifican relaciones de colaboración en la publicación de artículos científicos, la participación conjunta de especialistas de estas áreas del conocimiento en proyectos de investigación, resultados de tesis doctorales con enfoques interdisciplinares, el desarrollo de eventos científicos con temáticas afines, así como líneas de investigación que se identifican con la interacción de especialistas de varias áreas del conocimiento. Estas áreas representan los tipos de conocimiento que han sido generados, trasmitidos y divulgados como fase concluyente de la producción científica de la institución. De igual modo, estos conocimientos publicados son el producto natural e indispensable de la actividad científica por medio de la cual los investigadores garantizan la propiedad científica, el reconocimiento de una forma más amplia y la reafirmación de su reputación y la de la institución que representa (Piedra y Martínez, 2007).

El instrumento de medición integrado a la plataforma CV-UPR permite que los gestores realicen análisis más integrales, al contrastar los resultados de indicadores cuantitativos de las diferentes variables del proceso de ciencia y tecnología en diferentes rangos de tiempo. De esta forma se favorece el análisis de esta actividad en la institución de un modo flexible e integral. Las medidas cuantificadoras que provienen de la utilización del instrumento de medición precisan de una evaluación continua por parte de especialistas, y del análisis de los gestores de políticas científicas en la institución. De esta forma, los indicadores aportan información relevante para proyectar políticas para el incremento de la calidad de la investigación, la interacción de la institución con el entorno, la 
caracterización de la trayectoria científica del investigador, entre otros elementos que permiten determinar fortalezas y debilidades de la institución en materia de ciencia y tecnología.

\section{Conclusiones}

1. La utilización del curriculum vitae como fuente de información, así como el uso de los Sistemas de Información Curricular, como herramientas en la elaboración y aplicación de indicadores de medición de la gestión de la ciencia y la tecnología, se ha convertido en uno de los factores que favorecen la construcción de metodologías y procedimientos de medición, desde los preceptos de la bibliometría y la cienciometría.

2. El instrumento elaborado permite la caracterización de los investigadores, la producción científica y tecnológica, la trayectoria académica en relación con la investigación científica, la dinámica y la colaboración científica, y la visibilidad territorial e internacional; de esta forma se evidencia su enfoque abarcador en la medición de la ciencia y la tecnología a nivel institucional.

3. La presente investigación contribuye en la mejora del proceso de implementación del Sistema Integral para la Gestión de Información y Conocimiento Institucional (CV-UPR), como herramienta para la toma de decisiones de la institución objeto de estudio. Mediante la aplicación del instrumento de medición, los gestores de la ciencia y la tecnología de la institución obtienen una imagen sintética y contrastable de un grupo de variables que caracterizan el comportamiento de los principales procesos institucionales, lo que les permite tomar decisiones en esta actividad.

4. El instrumento propuesto, en interacción con las técnicas empíricas empleadas, ha permitido identificar los principales aspectos que caracterizan el desempeño del proceso de ciencia y tecnología en la Universidad de Pinar del Río, entre los cuales se encuentran:

- La institución posee investigadores con potencialidades reconocidas en altos grados académicos y científicos. Asimismo, existen políticas institucionales que favorecen la formación de especialistas, de forma gradual, y orientadas hacia la incorporación de jóvenes en la investigación científica para alcanzar grados científicos y realización profesional. Esta fortaleza de la institución constituye un factor importante para desarrollar la producción científica y tecnológica e incrementar la socialización del conocimiento científico.

- En el período de tiempo estudiado (2010-2014), la institución desarrolló sus capacidades de investigación con mayor fortaleza en las siguientes áreas del conocimiento científico: Ciencias forestales, Ciencias Económicas, Ciencias agrícolas, Pedagogía y Ciencias técnicas. En estas áreas se identifican los conocimientos de la institución que se encuentran arraigados y prevalecen en los resultados científicos y la actividad académica.

- La institución tiene visibilidad por la repercusión de sus resultados científicos en el desarrollo de la región circundante. De esta forma se comportan las relaciones de colaboración entre instituciones e investigadores en la producción científica, la 
actividad de proyectos de investigación, los convenios interinstitucionales, así como las asesorías y demás actividades académicas. Con respecto a la visibilidad de los resultados publicados en revistas científicas, se puede concluir que estos se publican en revistas indizadas en bases de datos de prestigio en Latinoamérica y en revistas nacionales de gran impacto en la región. No se puede decir lo mismo de la visibilidad en revistas internacionales, la cual se refleja en un grupo reducido de revistas de impacto.

\section{Referencias bibliográficas}

AENOR, Asociación Española de Normalización y Certificación (2003). UNE 66175. Norma Española. Sistemas de gestión de la calidad. Guía para la implantación de sistemas de indicadores. Madrid: AENOR.

Andreu, R., Ricart, J. E., y Valor, J. (1996). Estrategia y sistemas de Información. Madrid: McGraw Hill.

Arango Forero, G. (2013). Comunicación digital: una propuesta de análisis desde el pensamiento complejo. Palabra clave, 16(3), 673-697.

Arencibia, J. R. (2012). Sistematicidad en la evaluación de la actividad científica desde una perspectiva cienciométrica. Revista Acimed, 23(3), 215-218.

Armas, D., Díaz, M., y Giraldes, R. (2008). Sistema Institucional para la Gestión de la Ciencia y la Técnica en Universidades: una perspectiva cienciométrica para su análisis y evaluación. En Congreso Internacional de Información, La Habana, Cuba.

Barandiarán, S., y D'Onofrio, M. G. (2013). Construción y aplicación de una tipología de perfiles de diversidad profesional de los investigadores argantinos: aportes al Manual de Buenos Aires. En IX Congreso de Indicadores de Ciencia y Tecnología de la RICIYT, Bogotá, Colombia.

Barchini, G., Álvarez, M., Herrera, S., y Trejo, M. (2007). El rol de las ontologías en los sistemas de información. Revista de ingeniería informática, 14.

Calero, A. (1978). Técnicas de muestreo. La Habana: Editorial Pueblo y Educación.

Cañas, P., y Lorenzo, M. S. (2006). Sistemas de información científica: su aplicación al control de programas. En Taller Tecnimap, Sevilla, España.

Cañibano, C., Otamendi, J., y Solís, F. (2010). Investigación y movilidad internacional: análisis de las estancias en centros extranjeros de los investigadores andaluces. Revista española de documentación científica, 33(3), 428-457.

Catedral, V. J. (2012). Recomendaciones prácticas para la elaboración de indicadores de gestión de la salud y la seguridad en la construcción. En Taller COPARDOM para el Sector Construcción: aspectos laborales, seguridad social y prevención de riesgos, Santo Domingo, República Dominicana. 
CONEVAL, Consejo Nacional de Evaluación de la Política de desarrollo Nacional (2010). Guía para el diseño de Indicadores estratégicos. Madrid: Secretaría de Hacienda y Crédito Público.

D'Este, P., Castro, E., y Molas, J. (2009). Documento de base para un manual de Indicadores de Vinculación de la universidad con el entorno socioeconómico: un marco para la discusión. Buenos Aires: Observatorio Iberoamericano de la Ciencia, la Tecnología y la Sociedad.

D’Onofrio, M. G., Solís, F., Tignino, M. V., y Cabrera, E. (2010). Indicadores de trayectorias de los investigadores iberoamericanos: Avances del Manual de Buenos Aires y resultados de su validación técnica. Informe de la Red de Indicadores de Ciencia y Tecnología Iberoamericana e Interamericana. Buenos Aires: RICYT. Recuperado de http://www.oei.es/salactsi/ESTADO2010.pdf (Consultado el 12/04/2017)

Dietz, J. S., Chompalov, I., Bozeman, B., O'Neil L. E., y Park, J. (2000). Using the curriculum vitae to study the career paths of scientists and engineers: an exploratory assessment. Scientometrics, 49(3), 419-442.

Greco, C. (2012). Indicadores de la gestión universitaria. Herramientas para la gestión estratégica y la mejora de la calidad. Universidad Nacional de Quilmes, Argentina. En Indicadores universitarios: experiencias y desafíos internacionales. Santiago de Chile: Centro Interuniversitario de Desarrollo - CINDA.

Jaramillo, H., Lopera, C., y Albán, C. (2008). Carreras académicas. Utilización del CV para la modelación de carreras académicas y científicas. Bogotá: Facultad de Economía de la Universidad del Rosario.

Laudon, K. C. (2000). Administración de los sistemas de información. Madrid: Prentice Hall.

Mena, M. M. (2007). Utilidad de las soluciones archivísticas para la gestión de información en los sistemas electrónicos del sector de la salud. Revista Acimed, 15(3).

Miguel, S. E. (2011). Revistas y producción científica de América Latina y el Caribe: su visibilidad en SciELO, RedALyC y SCOPUS. Revista interamericana de bibliotecología, 34(2), 187-99.

Moreiro, J. A., Sánchez, S., y Morato, J. (2012). Mejora de la interoperabilidad semántica para la reutilización de contenidos mediante sistemas de organización del conocimiento. Revista eletrônica de biblioteconomia e ciência da informação, 33(17).

Navarrete, J., y Banqueri, J. (2008). Los sistemas de información científica: herramientas para medir el impacto de la investigación biomédica. Revista de medicina clínica (Barc), 131(Supl. 5), 71-80.

Nonaka, I. (1994). A dynamic theory of organizational knowledge creation. Organization science, 5(1), 14-37.

OCDE, Organización para la Cooperación y el Desarrollo Económico (1995). Manual on the measurement of human resources devoted to S\&T Canberra Manual. Paris: OCDE. 
OCDE, Organización para la Cooperación y el Desarrollo Económico (2003). Manual de Frascaty, 2002. Definiciones y convenciones básicas. Paris: OCDE. Recuperado de http://www.edutecne.utn.edu.ar/ocde/frascati-03-30-34.pdf (Consultado el 11/04/2017)

Piattini, M, G., Calvo Manzano, J. A., Cervera, J., y Fernández, L. (1996). Análisis y diseño de aplicaciones Informáticas de gestión. Madrid: Editorial Ra-Ma.

Piedra, Y. y Martínez, A. (2007). Producción científica. Ciencias de la información, 3(38), 33-38.

Ponjuán Dante, G. (2003). Gestión documental, de información y del conocimiento. Puntos de contacto y diferencias. Ciencias de la información, 34(3).

Ponjuán Dante, G., Mena, M., Villardefrancos, M. C., y León, M. (2004). Sistemas de información: conceptos y aplicaciones. La Habana: Félix Varela.

Reginaldo, Z. y González, A. (2012). Los indicadores ¿un invento desafortunado? Universidad de La Frontera. En Centro Interuniversitario de Desarrollo (2012). Indicadores universitarios: experiencias y desafíos internacionales. Santiago de Chile: Centro Interuniversitario de Desarrollo - CINDA.

RICYT, Red Iberoamericana de Indicadores de Ciencia y Tecnología (2007). Programa Iberoamericano de Ciencia y Tecnología para el Desarrollo (CYTED). Manual de indicadores de internacionalización de la ciencia y la tecnología, Manual de Santiago Recuperado de www.ricyt.org/manuales/doc view/1-manual-de-santiago (Consultado el 04/05/2017)

RICYT, Red Iberoamericana de Indicadores de Ciencia y Tecnología (2009). Manual de Lisboa. Pautas para la interpretación de los datos estadísticos disponibles y la construcción de indicadores referidos a la transición de Iberoamérica hacia la Sociedad de la Información. Recuperado de www.oei.es/historico/salactsi/manual_lisboa.pdf (Consultado el 04/05/2017)

Rivero Amador, S. (2011). La gestión de información, como dinamizadora de la gestión del conocimiento y de competencias en el ambiente organizacional. Revista COFIN, 4. Recuperado de http://www.cofinhab.uh.cu/index.php/RCCF/article/view/37 (Consultado el 04/05/2017)

Sebastián, J. (2011). Dimensiones y métrica de la internacionalización de las universidades. Universidades UDUAL, 51, 3-16. 